\title{
LOCO AT THE AUSTRALIAN SYNCHROTRON
}

\author{
M.J. Spencer, M.J. Boland, R.T. Dowd, G. LeBlanc, Y.E. Tan, ASP, Melbourne, Australia
}

\section{Abstract}

LOCO has been used during the commissioning of the Australian Synchrotron storage ring with a number of benefits. The LOCO (linear optics from close orbits) method compares a model response matrix to the real machine response matrix. Using this approach we are able to adjust the machine to match the ideal model. Results presented here show that LOCO has provided a high degree of control over a wide range of machine parameters.

\section{INTRODUCTION AND THEORY}

The LOCO method uses a measured response matrix to calibrate a computer model [1]. Certain fit parameters in the model are changed in order to minimise the difference between the model's response matrix and the machine's response matrix. Typically, the parameters used to calibrate the model are:

- $\quad$ BPM gains and coupling

- $\quad$ Corrector magnet gains and coupling

- Quadrupole strengths

- $\quad$ Skew quadrupole strengths

The initial model is set up to have the desired beam qualities. The changes made to calibrate this ideal model can then be applied to the machine in reverse. The machine's behaviour should then, in principle, be the same as the ideal model. This approach requires that you chose fit parameters which are also easily varied in the real machine.

For a detailed outline of the Australian Synchrotron lattice see [2].

\section{BPMS AND CORRECTORS}

\section{Gains}

The LOCO calibrated model shows a $4 \%$ variation in the BPM gains (fig 1). This is most likely caused by differences in the electrical characteristics of the BPM buttons and cables.

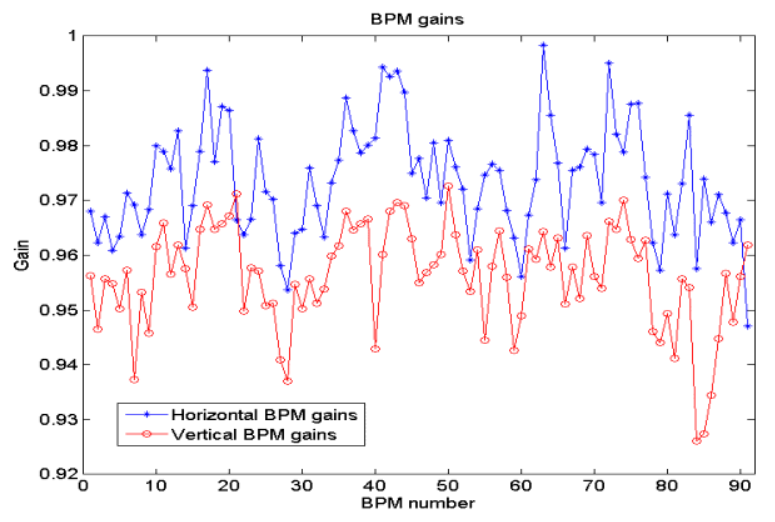

Figure 1: BPM gains.
There is a $1-2 \%$ variation in the horizontal corrector magnet strengths, and a 3-4\% variation in the vertical magnet strengths (fig 2).

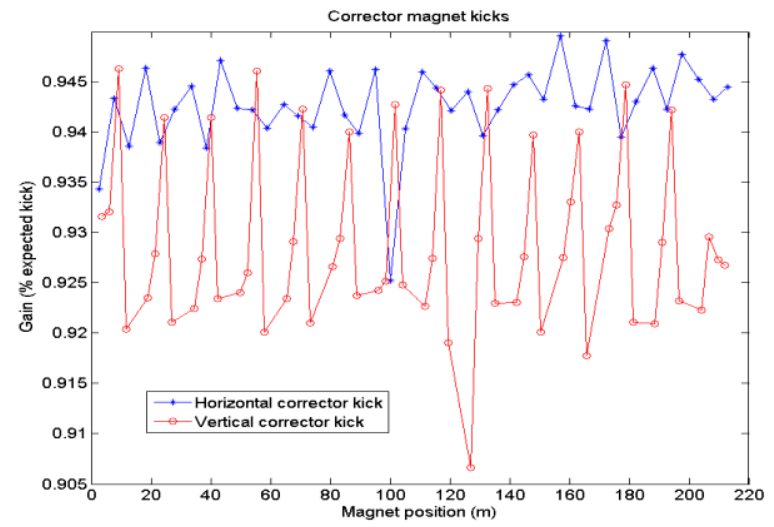

Figure 2: Corrector magnet strengths.

The regular pattern seen in the corrector gains is most likely due to irregularities in the sextupole corrector windings.

\section{Coupling}

LOCO gives two coupling values for each BPM block: $\mathrm{x}$-coupling and y-coupling (fig 3).

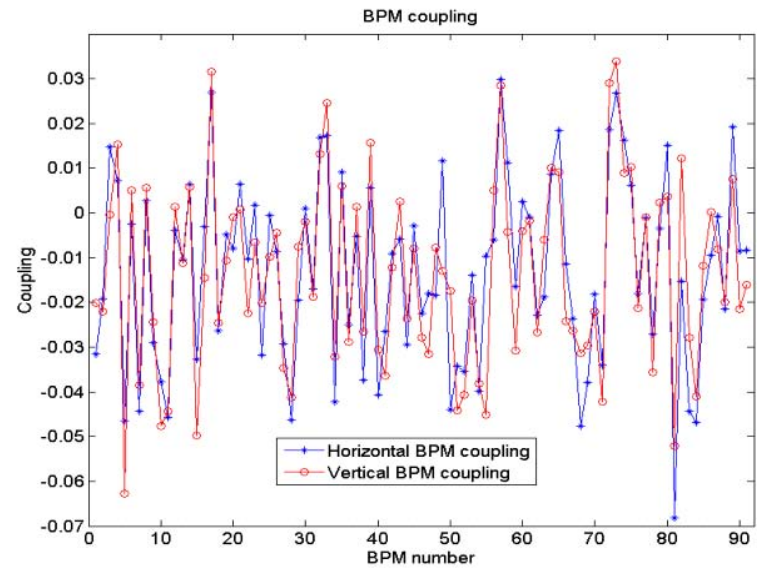

Figure 3: BPM coupling.

The BPM coupling appears to be less than $4 \%$ on average but up to $6 \%$ in some cases. It was confirmed that the block itself is not rotated by this much. If it is due to BPM block rotation then the ratio of the two coupling values should be -1 . However figure 3 shows that the ratio is on average +1 . This implies that the coupling is due to differences in the cable attenuations between buttons. [3]

Figure 4 shows that the corrector magnet coupling averages less than $0.5 \%$. The combined BPM and corrector coupling factors are consistent with direct observation of the orbit change with corrector change. 


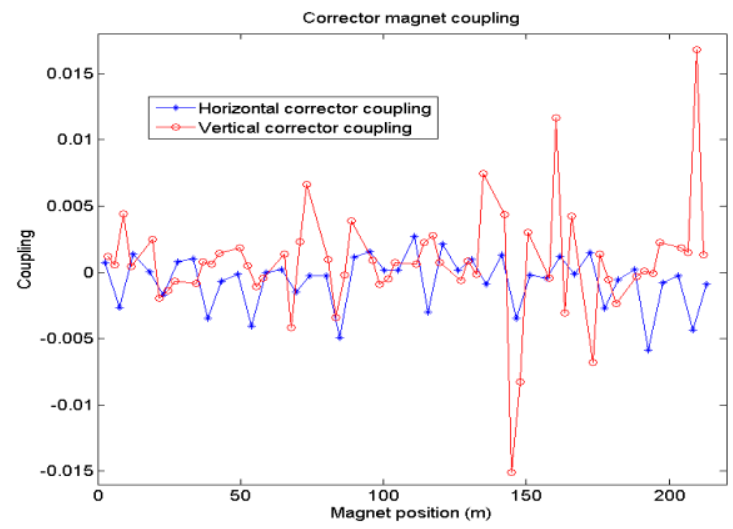

Figure 4: Corrector magnet coupling.

\section{QUADRUPOLES, BETA FUNCTIONS AND TUNES}

A calibrated model was created from the un-corrected machine. The beta functions are shown in figures 5 and 6 .

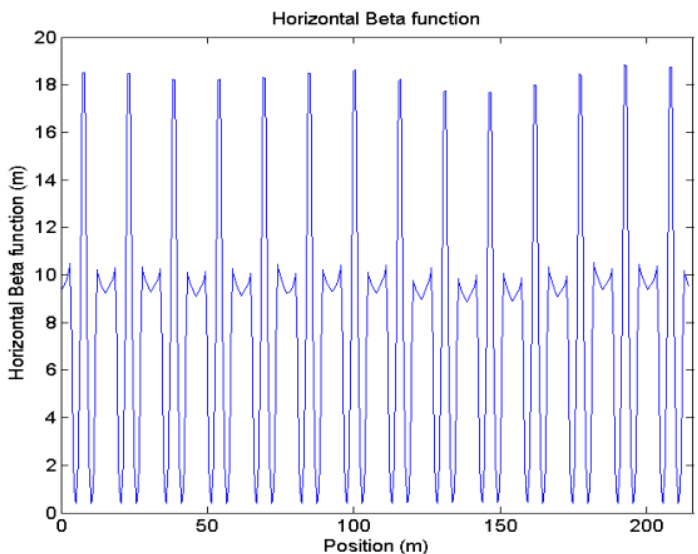

Figure 5: Initial horizontal beta function. (calibrated model)

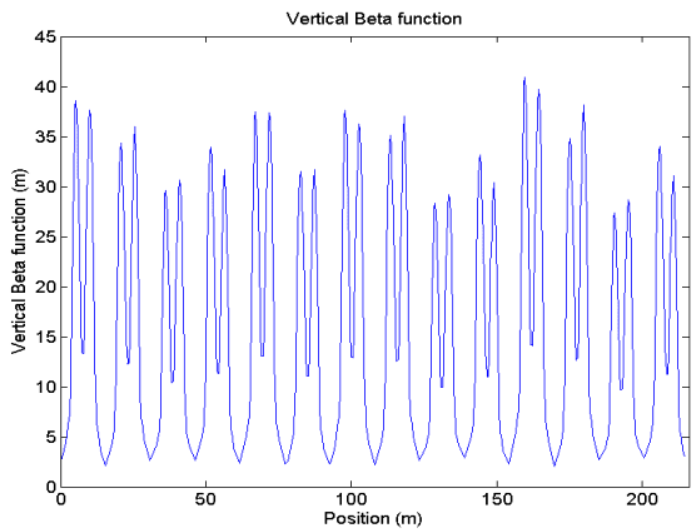

Figure 6: Initial vertical beta function. (calibrated model)

Figure 7 shows the settings of the 3 quadrupole families that were found to restore the ring symmetry.
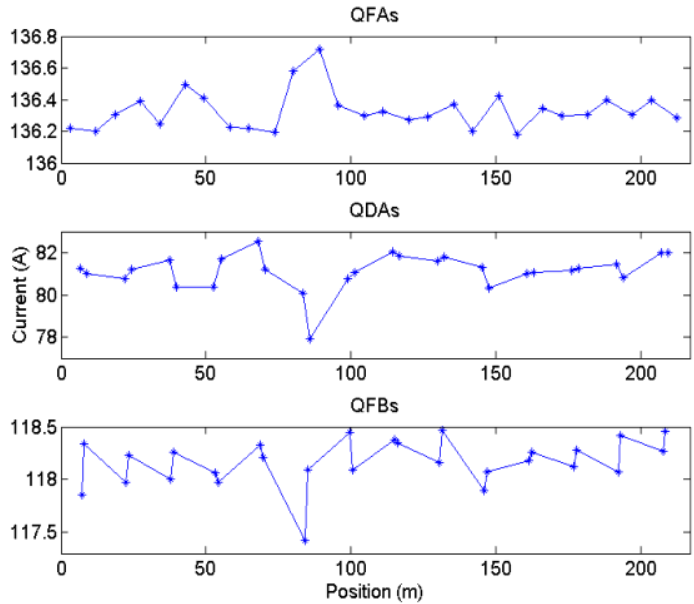

Figure 7: Quadrupoles settings used to restore ring symmetry.

A calibrated model was created from the machine once the quadrupoles were set in this way. Figure 8 and 9 shows that the ring symmetry has now been restored

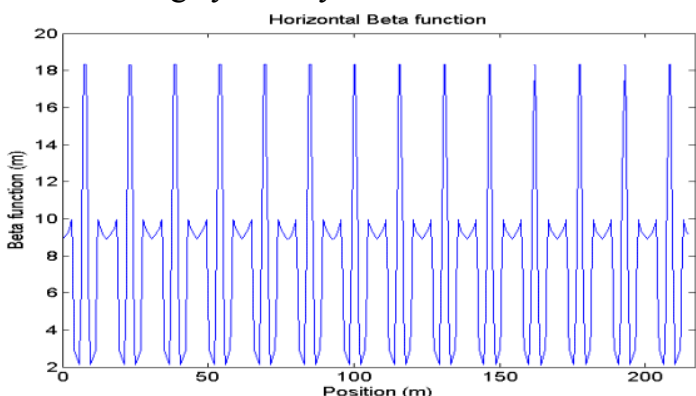

Figure 8: Corrected machine horizontal beta function. (calibrated model)

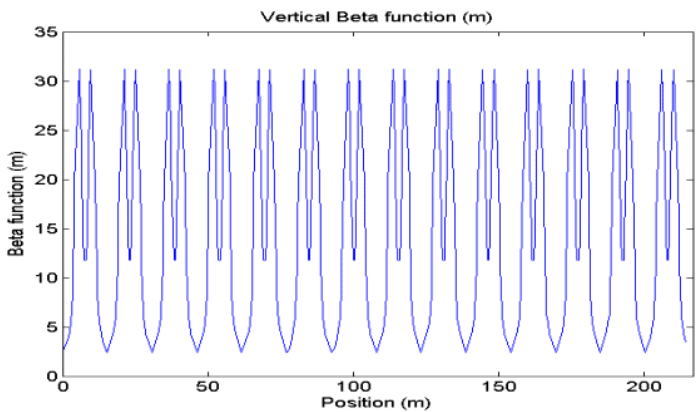

Figure 9: Corrected machine vertical beta function (calibrated model)

Table 1 compares the ideal model tune, the measured machine tune, and the calibrated model tune.

Table 1: The tunes from the ideal model, calibrated model, and real machine.

\begin{tabular}{|c|c|c|}
\hline & $\mathrm{x}$-tune & $\mathrm{y}$-tune \\
\hline Ideal model & 13.29000 & 5.21600 \\
\hline $\begin{array}{c}\text { Real machine } \\
\text { (measured after } \\
\text { correction) }\end{array}$ & 13.2919 & 5.2177 \\
\hline Calibrated model & 13.29007 & 5.21598 \\
\hline
\end{tabular}

D01 Beam Optics - Lattices, Correction Schemes, Transport

1-4244-0917-9/07/\$25.00 (c)2007 IEEE 


\section{ZERO DISPERSION AND DISTRIBUTED DISPERSION LATTICES}

Initially the machine was set in zero dispersion mode (dispersion $=0 \mathrm{~m}$ in the straight sections). Figure 10 compares the ideal model's horizontal dispersion with the measured dispersion of the corrected machine.

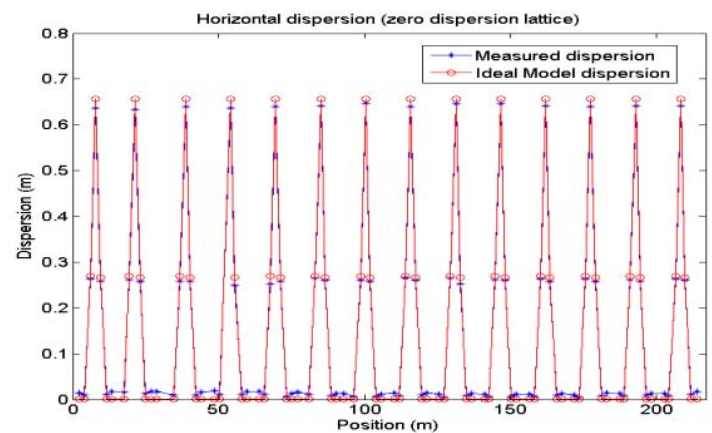

Figure 10: Model and machine horizontal dispersion. (zero dispersion)

A new ideal model was created with distributed dispersion (dispersion $=0.1 \mathrm{~m}$ in the straight sections). This model was used to correct the machine to a distributed dispersion configuration. Figure 11 compares the model's horizontal dispersion with the measured dispersion of the corrected machine.

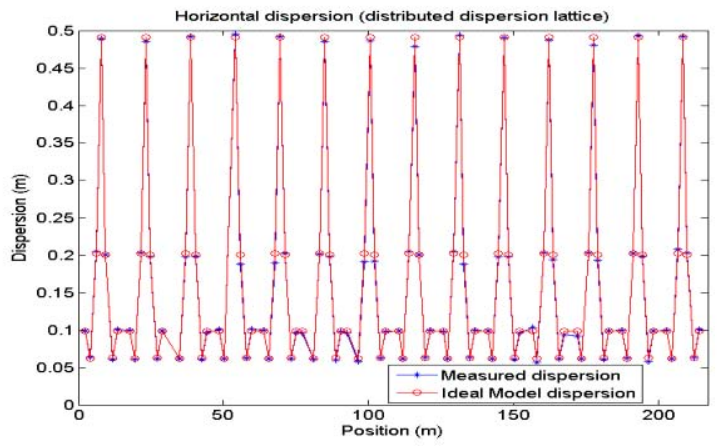

Figure 11: Model and machine horizontal dispersion. (distributed dispersion)

\section{SKEW QUADRUPOLES}

Figure 12 shows the strengths of the skew quadrupoles in the calibrated model. These values are the negative of the values which should be applied to the machine to remove all coupling errors.

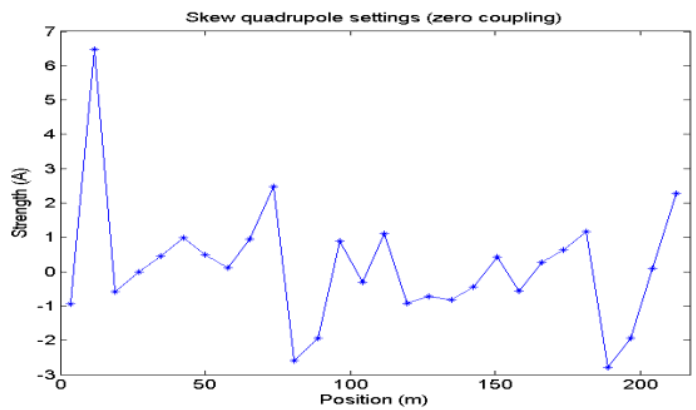

Figure 12: Skew quadrupole strengths in the calibrated model.

\section{SINGULAR VALUE SELECTION}

Initially, all but the smallest singular values were used during calculations. This resulted in a divergence of the strengths of the QD magnets in the calibrated model. Removing the final 30 singular values has become the normal approach to ensure a realistic calibrated model (fig 13)

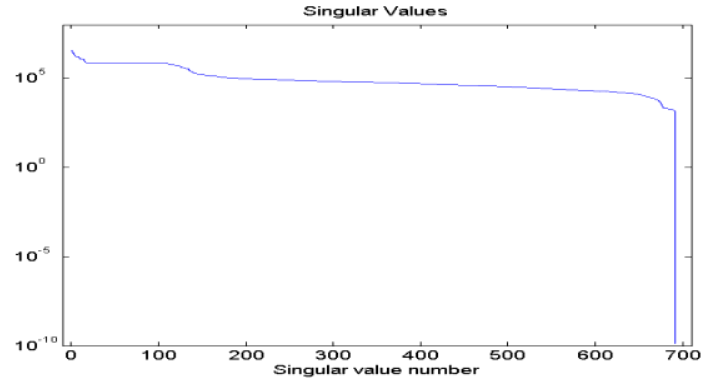

Figure 13: A typical plot of the initial singular values.

\section{DISCUSSION AND CONCLUSION}

LOCO has been a powerful tool during the commissioning of the Australian Synchrotron. It has been used to:

- $\quad$ Correct the beta functions.

- Introduce arbitrary dispersion in the straight sections.

- Calculate optics compensation for insertion devices

- Correct the coupling.

- Analyse the coupling present in the BPMs.

- Accurately model the tune to compare with the measured tune.

In the future it is intended to

- Attempt to gang the central QF magnets to prevent divergence in their strengths.

- Model all magnet rotations and translations including the dipole magnet (which includes its quadrupole component).

- Completely calibrate a model with all possible fit parameters to be used as a base model for future LOCO runs.

- Investigate possible methods of singular value selection and how it relates to divergence in the fit parameter families.

\section{REFERENCES}

[1] J. Safranek, "Experimental determination of storage ring optics using orbit response measurements", Nuclear Instrument and Methods, 388 (1997), 27-36.

[2] J.W. Boldeman, D. Einfeld, „The physics design of the Australian synchrotron storage ring", Nuclear Instruments and Methods, 521 (2004), 306-307.

[3] Y.E. Tan, "Storage Ring Turn-By-Turn BPMs At The Australian Synchrotron", these proceedings. 